\title{
TRADICIONES LÚDICAS EN EL CAMINO CENTRAL PORTUGUÉS: EL JUEGO DE BOLOS EN LA PARTE FINAL DEL CAMINO DE SANTIAGO
}

\author{
TRADIÇÕES RECREATIVAS NO CAMINHO CENTRAL PORTUGUÊS: O JOGO \\ DE BOLOS NO FINAL DO CAMINHO DE SANTIAGO
}

\author{
PLAYFUL TRADITIONS IN THE CENTRAL PORTUGUESE WAY OF ST. JAMES: \\ BOWLING AT THE END OF THE WAY
}

\author{
José Eugenio Rodríguez Fernández*, Beatriz Oliveira Pereira**, \\ Joaquín Lago Ballesteros*
}

\begin{abstract}
Palabras clave:
Actividades

recreativas.

Juegos

recreacionales.

Bolos.

Cultura popular.

Resumen: Este estudio buscó determinar la existencia del juego de bolos tradicional en la parte final del Camino Central Portugués de Santiago (Oporto-Valença do Minho), hecho que nos permitió continuar con el estudio realizado en Galicia en el Camino de Santiago y que nos permite comprender la génesis de este juego popular y tradicional. Se realizó un estudio cualitativo, descriptivo e interpretativo, utilizando la entrevista como herramienta para la obtención de datos. La muestra del estudio estuvo formada por 27 personas: nueve técnicos deportivos de Cámaras Municipales, nueve docentes especialistas en Educación Física (EB2) y nueve docentes no especialistas en Educación Física (EB1). Se registró una escasa práctica del juego de bolos tradicional en los municipios por donde pasa el Camino Central Portugués, a excepción del municipio de Paredes de Coura, donde el Jogo do Chavelho se muestra como una modalidad exclusiva de esta localidad portuguesa.
\end{abstract} Atividades de lazer. Jogos recreativos. Bolos.

Cultura popular.
Palavras chave:

Resumo: Este estudo procurou determinar a existência da modalidade de bolos tradicional na parte final do Caminho Central Português de Santiago (Porto-Valença do Minho), fato que nos permitiu continuar com o estudo realizado na Galiza no Caminho de Santiago e que nos permite compreender a gênese deste jogo popular e tradicional. Foi realizado um estudo qualitativo, descritivo e interpretativo, que usou a entrevista como uma ferramenta para obter dados. A amostra do estudo foi composta por 27 pessoas: nove técnicos do desporto das Câmaras Municipais, nove professores especialistas em Educação Física (EB2) e nove professores não-especialistas em Educação Física (EB1). Foi registrada uma reduzida prática do jogo de bolos tradicional nos municípios onde passa o Caminho Central Português, com exceção do município de Paredes de Coura, mostrando o Jogo do Chavelho como uma forma única de prática nesta cidade portuguesa.

\section{Keywords:}

Leisure activities

Recreational games. Bowling.

Popular culture.
Abstract: This study sought to determine the existence of traditional bowling (Juego de Bolos) in the final part of the Central Portuguese Way of St. James (Porto-Valença do Minho), which would allow us to continue the study conducted in Galicia's Way of St. James to understand the genesis of that popular and traditional game. We conducted a qualitative, descriptive and interpretive study using interviews as a tool for data collection. The study's sample included 27 people: nine Municipal sports coaches, nine teachers specialized in Physical Education (EB2), and nine non-specialist Physical Education (EB1) teachers. There was little practice of traditional bowling in the municipalities crossed by the Central Portuguese way, with the exception of Paredes de Coura, where the Chavelho's game was unique to that Portuguese town.
*Universidade de Santiago de Compostela. Santiago de Compostela, España.

E-mail: geno.rodriguez@usc.es; joaquin.lago@usc.es

**Universidade do Minho. Braga. Portugal.

E-mail: beatriz@ie.uminho.pt

Recebido em: 24-07-2017 Aprovado em: 21-05-2018

DOI: http://dx.doi.org/10.22456/1982-8918.75202 (c) (1) () Licence 


\section{INTRODUCCIÓN}

El juego es una de las expresiones más comunes del ser humano, una parte esencial de la naturaleza humana y que está presente en nuestra cultura (ARAÚJO et al., 2013) desde las sociedades más antiguas. Las características intrínsecas del juego nos muestran una actividad placentera y socializadora (RODRÍGUEZ; PAZOS; PALACIOS, 2014a), con un fin en sí misma, donde a través de su práctica se pueden adquirir una serie de valores (ANDREU, 2009; REBOLLO, 2002; LAVEGA; NAVARRO, 2015; MÉNDEZ-GIMÉNEZ; FERNÁNDEZRíO, 2011) que posibilitarán el desarrollo integral de la persona en los ámbitos social, cultural, educativo, afectivo o físico (LARA, 2011).

Numerosos autores relacionan el juego y cultura (LAVEGA; NAVARRO, 2015; PARLEBAS, 2001; RODRíGUEZ, 2016; RUIZ, 2000), un binomio inseparable si lo consideramos como una actividad que refleja la forma de ser de la gente (VIGNE, 2011), de los pueblos que habitan, de las costumbres del lugar y que, de cierta forma, nos sirve para interpretar la evolución de la humanidad desde una perspectiva lúdica, natural y adaptada a la realidad. Parlebas (2013) destaca en este sentido que el juego es el espejo de la sociedad, imagen viva de la comunidad marcada por las acciones de sus habitantes y, como forma parte de la cultura, se puede considerar como uno de los elementos del patrimonio inmaterial de una sociedad (NASCIMENTO et al., 2015). De hecho, en el año 2010 la Unesco decidió inscribir los juegos populares y tradicionales en la lista de prácticas del patrimonio cultural mundial (PARLEBAS, 2013).

Nos referimos entonces a los juegos populares y tradicionales porque son los que mejor contribuyen a esa relación con la sociedad y la cultura (RODRIGUEZ, 2013), esa tipología de juegos que por su relación con el mundo laboral (CABRAL, 1998; DIAS; MENDES, 2010; VEIGA, 1998) han ido mostrando las peculiaridades de cada época histórica de una forma singular.

Rodríguez (2016) matiza los términos asociados a la palabra juego, popular (manifestación lúdica arraigada en una sociedad, donde gran cantidad de personas lo practican) y tradicional (prácticas que han llegado a nuestros días transmitidos de generación en generación a través de la oralidad o la práctica viva), aunque habitualmente suelen utilizarse indistintamente (juntos o por separado) para referirse al mismo concepto, aunque su esencia nos lleva a diferentes interpretaciones. Indica además el autor que este tipo de juegos se encuentran en un momento de total abandono, una situación que nos muestra la necesidad de realizar acciones encaminadas a su promoción y recuperación, sobre todo, en el ámbito social y educativo.

Parlebas (2013, p.11) profundizaba en este aspecto haciendo referencia a que "[...] hoy, el gran viento de la modernidad, barre como un tornado los juegos tradicionales. La ruptura tecnológica y cultural que conoce el conjunto de las sociedades actuales tiende a provocar el abandono de costumbres antiguas y a substituir prácticas del pasado por nuevos comportamientos" y que "[...] los juegos tradicionales no solamente están cada vez más abandonados, sino que también son calificados como juegos inferiores [...]"

Sin embargo, algunos de los juegos populares y tradicionales más importantes y más practicados hoy en día es posible que consiguieran sobrevivir a los aires de modernización de la sociedad (también en el campo de la actividad física y el deporte) fruto de un proceso de deportivización (MÉNDEZ-GIMÉNEZ; FERNÁNDEZ-RíO, 2011; PARLEBAS, 2001; 
RODRÍGUEZ, 2016; RUIZ, 2000). Se trata de juegos en esencia tradicionales (práctica libre, reglas flexibles, adaptables al participante y al entorno...) pero que estandarizaron reglas básicas para convertir estas prácticas en deportes propiamente dichos, donde prima la competición y el espectáculo.

Es el caso del juego de bolos, posiblemente uno de los más practicados en el mundo en sus diferentes modalidades (RODRIGUEZ; PAZOS; PALACIOS, 2014b; RUIZ, 2000). Rodríguez De La Cruz (2008) destaca que es el juego popular y tradicional del que más información escrita disponemos, una modalidad de lanzamiento que exige altas dosis de precisión y, dependiendo de la modalidad, también de fuerza (ALONSO et al., 2015). RODRIGUEZ (2016) y RUIZ (2000) apuntan en este sentido que ambas habilidades (precisión y fuerza) caracterizan las dos grandes familias de juego de bolos: de derribo (donde el objetivo consiste en derribar únicamente los bolos y, según del número de derribos, se establece la puntuación) y pasabolos (que consiste en derribar los bolos y desplazarlos lo más lejos posible y, según la distancia alcanzada por los bolos, se establece la pertinente puntuación).

Como muchos juegos populares y tradicionales, los bolos es una modalidad típica de hombres adultos, que margina de cierta forma el universo lúdico femenino (HERNÁNDEZ et al., 2015; RODRÍGUEZ; PAZOS; PALACIOS, 2016), aunque se tenga constancia de modalidades de bolos practicadas sólo por mujeres en regiones españolas como Aragón, que "ejemplifica muchas de las características de los juegos femeninos practicados en la sociedad rural tradicional: transmisión oral de madres a hijas, es una práctica ritualizada, aculturación en los roles de género o formas de uso del espacio público por las mujeres" (PONTES, 2014, p. 85).

En España, está documentada una estrecha relación entre el Camino de Santiago y este juego milenario, como un legado o influencia de los peregrinos que recorrían esta vía espiritual y cultural hacia Santiago de Compostela por los diferentes caminos o vías registrados desde hace años (RODRIGUEZ; PAZOS; PALACIOS, 2014b; RODRIGUEZ; PEREIRA, 2016; RUIZ, 2000; TORRE, 2013).

Según Ramos (2011, p. 8): "[...] el Camino de Santiago puede considerarse la primera vía cultural europea (con el inevitable trasvase de ideas) y, tal vez, la más importante por su pervivencia centenaria y por su papel religioso, histórico, literario y artístico, vigente en la actualidad". Añade que la peregrinación ha sido la corriente europea actuando sobre nuestra historia y sobre nuestra cultura, una vía de relaciones personales, de intercambio de ideas y corrientes artísticas y culturales. De las ocho vías que se dirigen a Santiago de Compostela (Vía de La Plata, Camino Francés, Camino Primitivo, Camino del Norte, Camino Inglés, Camino de Fisterra, Ruta del Mar de Arousa y Camino Portugués), este último, el Camino Central Portugués, es uno de los más importantes (POMBO, 2012; SÁNCHEZ, 2010) y, detrás del Camino Francés, el que más afluencia tiene hoy en día.

Rodríguez, Pazos y Palacios (2014b) señalan que en las vías de entrada en Galicia (España) por los Caminos de Santiago, se registra una importante actividad del juego de bolos tradicional, sobre todo, en la zona sur de Galicia, lugar que coincide con la entrada en España del Camino Central Portugués. No se tienen datos relativos a la práctica de esta modalidad en territorio lusitano, pero si de otro tipo de juegos populares y tradicionales como puede ser el ejemplo del "Jogo do Beto" (ARAÚJO et al., 2013) o del propio "Jogo da Malha" (NASCIMENTO et al., 2015; RIBAS, 2013), el juego popular y tradicional con más arraigo en la zona norte de Portugal (CABRAL, 1998; RODRÍGUEZ; PEREIRA, 2016). 
Y es que Portugal, país con una enorme riqueza cultural, la tradición lúdica y, en concreto, el juego popular y tradicional, cuenta con multitud de manifestaciones vinculadas a la forma de vida de sus gentes (SILVA, 2011; SILVEIRA; CUNHA, 2014); manifestaciones que, por desgracia, cada vez son menos frecuentes y en muchos casos, pasarán al olvido si no se realiza un programa serio de recuperación y promoción de este tipo de prácticas.

Nuestra hipótesis de partida con esta investigación es que, si en el Camino Portugués que discurre por Galicia, se registra una gran actividad del juego de bolos tradicional, es posible que en la continuación de esta vía en tierras portuguesas se registre también una actividad similar, sobre todo, en las zonas más cercanas a Galicia.

Por lo tanto, nuestro objetivo es conocer y registrar las modalidades de juego de bolos que se manifiestan en el Camino Central Portugués, en la zona norte, que comprende 9 municipios (de Porto a Valença do Minho).

\section{MATERIAL Y MÉTODO}

\subsection{Diseño}

Se realizó un estudio cualitativo como estrategia metodológica (DENZIN; LINCOLN, 2015) encuadrado bajo un paradigma descriptivo e interpretativo (AMADO, 2014; LIMA; PALHARES, 2014). Se optó por este tipo de metodología por la importancia del contexto y sus interrelaciones con los participantes en el estudio (FLICK, 2015) y, sobre todo, porque nos acerca más a la realidad y a la vida real de la gente, que esconde en muchas ocasiones verdaderas historias relacionadas con la cultura y las tradiciones, es decir, el objeto de nuestra investigación.

El estudio se centró en el tramo del Camino Central Portugués del Camino de Santiago que discurre entre las localidades de Porto y Valença do Minho, un recorrido de 125 kilómetros que atraviesa nueve municipios: Valença do Minho, Paredes de Coura, Ponte de Lima, Barcelos, Póvoa de Varzím, Vila do Conde, Maia, Matosinhos y Porto (ver figura 1). Se realizaron asimismo comparativas con la zona norte de Portugal y con el tramo final del Camino Portugués que discurre por tierras gallegas.

Figura 1 - Mapa de la zona norte de Portugal, con sus correspondientes distritos. A la izquierda se destacan los municipios por donde discurre el Camino Central Portugués (Porto-Valença do Minho).

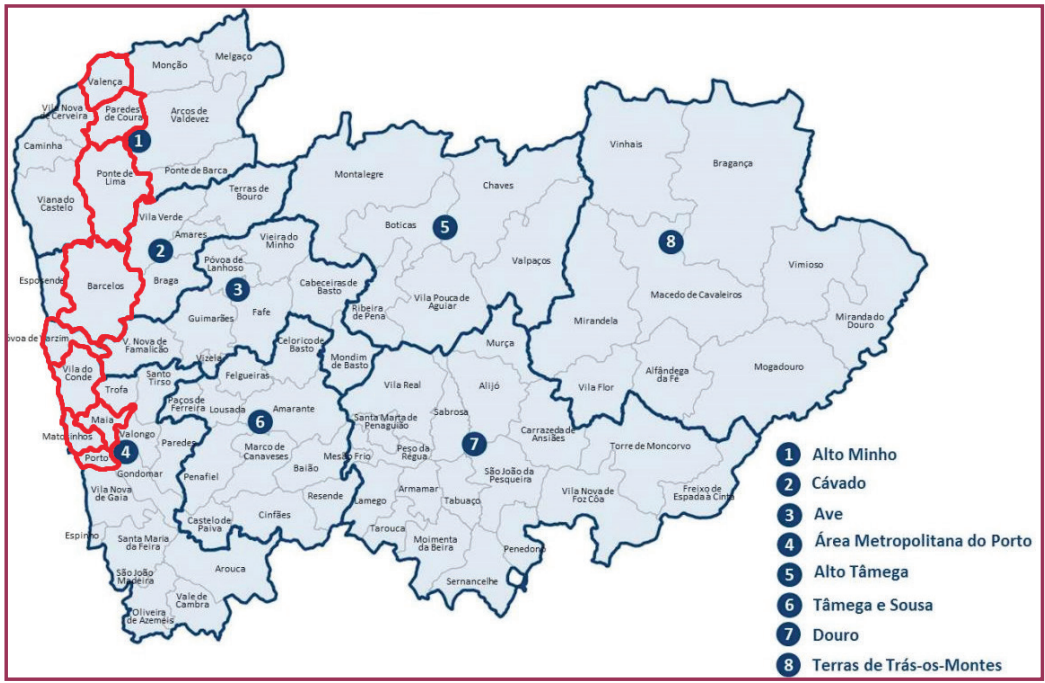

Fuente: CCDRN-Comissão de Coordenação e Desenvolvimento Regional do Norte (www.ccdr-n.pt) 


\subsection{Muestra}

Participaron en el estudio un total de 27 personas relativas a dos ámbitos de trabajo diferentes: gestión deportiva municipal y docencia. En cada uno de los nueve municipios analizados se entrevistó a uno de los técnicos deportivos municipales y dos profesores: uno de primer ciclo de escuela básica (EB1, que impartían docencia del área pero no tenían que ser necesariamente especialistas en Educación Física) y uno del segundo ciclo de escuela básica (EB2, que eran especialistas en Educación Física e impartían materias específicas del área en el centro escolar). Así la muestra estaba formada por 9 técnicos deportivos municipales, 9 docentes de EB1 y 9 docentes de EB2.

\subsection{Instrumento}

Se utilizó la entrevista semiestructurada como herramienta metodológica para la obtención de datos (BAPTISTA, 2016; BOGDAN; BIKLEN, 2013; QUIVY; CAMPENHOUDT, 2017). Una vez elaborada la entrevista y las correspondientes preguntas, fue sometida a un juicio de expertos universitarios (profesores) para su validación, un método útil para verificar su fiabilidad, definida por Escobar-Pérez y Cuervo-Martínez (2008, p. 29) como "una opinión informada de personas con trayectoria en el tema, que son reconocidas por otros como expertos cualificados en éste, y que pueden dar información, evidencia, juicios y valoraciones".

Este juicio de expertos proporcionó información (claridad de las preguntas, relevancia, número, tiempo para contestar, uso apropiado de palabras, formato de la entrevista...) para mejorar el instrumento de recogida de datos, para que cumpliera con el propósito y objetivo del estudio. En base al conjunto de recomendaciones realizadas por los expertos, se revisó nuevamente el instrumento y se realizaron los ajustes pertinentes.

Las entrevistas fueron realizadas por un único investigador, en el centro de trabajo de cada uno y grabada en audio. Asimismo, se recurrió a la revisión de documentos escritos (trabajos científicos, publicaciones...) para comprender mejor la realidad estudiada e incrementar la información pertinente para el estudio.

\subsection{Procedimiento}

Para la realización del estudio se tuvieron en cuenta una serie de pautas y actuaciones distribuidas temporalmente con el objetivo de dotar la investigación de una organización eficaz. En primer lugar, se solicitó permiso a la Comissão de Ética da Universidade do Minho (CEUM). Recibida la autorización se procedió con el desarrollo del estudio:

- Se contactó con los informantes, primero por correo electrónico y, posteriormente, en persona.

- De esta primera reunión el investigador decidió si era un informante válido para participar en el estudio. Se barajaron los siguientes criterios de exclusión:

- Que los informantes llevaran más de tres años en ese puesto de trabajo.

- Que fueran conocedores de la realidad social del municipio donde trabajaban.

- Que estuvieran en contacto frecuente con la organización y/o desarrollo de actividades de ocio, juego, tiempo libre (técnicos deportivos municipales) y que impartieran materias 
de Educación Física en la escuela (docentes).

- Se informó a los participantes de los objetivos del estudio, las personas y entidades que en él intervenían y se les solicitó permiso para realizar la entrevista y grabarla en audio.

- Se realizó la entrevista en el lugar de trabajo de cada informante, con el fin de facilitar su participación, disponer de un lugar tranquilo e invertir el tiempo justo en este estudio. La duración media de cada entrevista fue de 20 minutos.

- Durante las entrevistas el investigador tomó notas en el cuaderno de campo.

- En cada municipio se entrevistó primero al técnico deportivo y, posteriormente, a los profesores de EB1 y EB2. El orden de los municipios analizados fue el siguiente: Valença do Minho, Paredes de Coura, Ponte da Lima, Barcelos, Póvoa de Varzím, Vila do Conde, Maia, Matosinhos, Porto.

- Se realizó la transcripción de las entrevistas y, siguiendo las normas éticas del estudio, realizado este proceso se procedió con la destrucción de las mismas.

- El análisis de la información se realizó empleando las herramientas del paquete office de Microsoft, no recurriendo a ningún programa específico de análisis cualitativo por la baja complejidad de las relaciones y por el carácter altamente descriptivo del estudio. La credibilidad y validez de la información proporcionada se constató, fundamentalmente, por la visita del investigador al lugar de los hechos y contrastando la información con otras fuentes (Federación Gallega de Bolos).

\section{RESULTADOS}

Después del análisis de las entrevistas y la documentación revisada, se constató que el juego de bolos tradicional no es una actividad practicada habitualmente en los municipios por los que transcurre el Camino Portugués (de Porto a Valença do Minho). La extraordinaria actividad registrada en el Camino Portugués en la zona sur de Galicia no encuentra continuidad a su entrada en el país luso. La tradición lúdica en esta zona de Portugal está marcada por el juego de "A Malha1", referido anteriormente por Cabral (1998) y Rodríguez y Pereira (2016) como el juego popular y tradicional con más arraigo en la zona norte de Portugal. En la tabla 1 podemos observar los juegos populares y tradicionales de adultos más practicados en esta zona del camino estudiada.

Tabla 1 - Juegos populares de adultos más practicados en el tramo Porto-Valença do Minho.

\begin{tabular}{lccc}
\hline \multicolumn{4}{c}{ Juegos populares y tradicionales de adultos } \\
\hline Municipio & Jogo da Malha & Juego de bolos & Juego de Petanca $^{2}$ \\
\hline Valença do Minho & $\sqrt{ }$ & - & $\sqrt{ }$ \\
Paredes de Coura & $\sqrt{ }$ & $\sqrt{ }$ & $\sqrt{ }$ \\
Ponte de Lima & $\sqrt{ }$ & - & $\sqrt{ }$ \\
Barcelos & $\sqrt{ }$ & - & $\sqrt{ }$ \\
Póvoa de Varzím & $\sqrt{ }$ & - & $\sqrt{ }$ \\
Vila do Conde & $\sqrt{ }$ & - & $\sqrt{ }$ \\
Maia & $\sqrt{ }$ & - & $\sqrt{ }$ \\
Matosinhos & $\sqrt{ }$ & & \\
Porto & $\sqrt{c}$ Fuente: elaboración propia.
\end{tabular}

1 Jogo da malha. En un terreno liso y plano se colocan dos cilindros de madera (pinos) de $18 \mathrm{~cm}$. de alto y $6 \mathrm{~cm}$ de diámetro, separados entre 
En el municipio de Paredes de Coura nos encontramos con el dato de la práctica de la modalidad de juego de bolos tradicional. Nuestra investigación nos muestra que en esta localidad portuguesa se practica el Jogo tradicional do Chavelho, una modalidad de bolos única que se caracteriza por:

- Historia. Es un juego típico y con larga tradición en el municipio de Paredes de Coura (Portugal). Antigüamente se jugaba en los 21 barrios en los que se organiza el ayuntamiento, principalmente los domingos y festivos, en lugares estratégicos como podían ser cerca de la iglesia o en el centro de la localidad. El trabajo de promoción desarrollado por el Servicio Municipal de Deportes, en colaboración con Asociaciones Deportivas y Centros de Enseñanza de la localidad, ha permitido que esta práctica ancestral siga viva en este municipio portugués, considerándose el jogo tradicional do Chavelho es una marca indeleble del área sociocultural, deportiva y recreativa del Ayuntamiento de Paredes de Coura, en el que se intenta promover su divulgación en eventos con gran afluencia de gente (como pueden ser el Encontro Nacional de Jogos Tradicionais em Santarém ou en los Jogos do Futuro organizados por la Câmara Municipal de Lisboa).

- Campo de juego. Se practica en una superficie llana, generalmente de barro o tierra, con unas distancias aproximadas de 30 metros de largo y 5 metros de ancho. Aunque se puede practicar en cualquier superficie que reúna estas características, en el municipio de Paredes de Coura cuentan con espacios construidos específicamente para este fin (ver figura 2).

Figura 2 - Campo para el Jogo do Chavelho situado

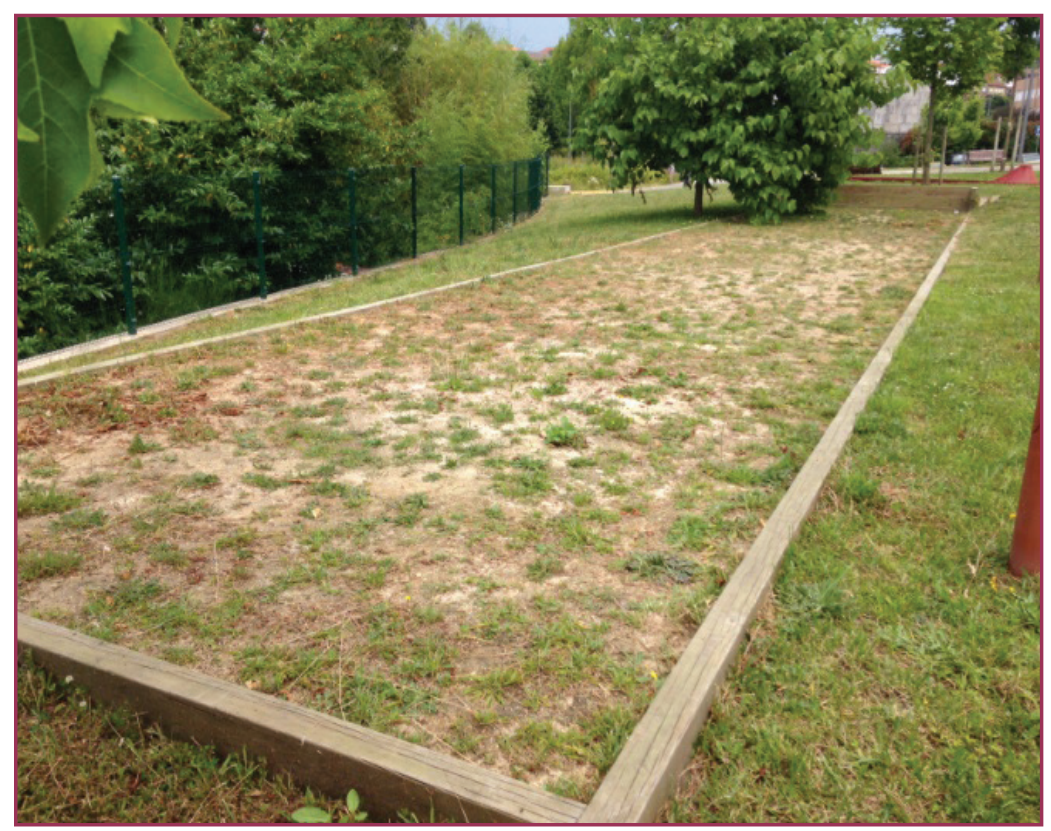

Fuente: Elaboración propia.

- Elementos de juego. La bola de lanzamiento mide cerca de 12 centímetros de diámetro y pesa aproximadamente 1,5 kilogramos (los jugadores se refieren a ella como un queijo limiano, en referencia a una bola de queso típica de esta región). Los 14 Chavelhos de juego tienen las siguientes medidas:

a) 10 cos de 20 centímetros de altura.

b) 1 Chavelho de 30 centímetros de altura.

2 Juego de Petanca. En un terreno llano, normalmente arenoso, de unas dimensiones aproximadas de 15x4 metros, un jugador lanza con la mano una bola pequeña (boliche), con ambos pies en el suelo y en posición estática desde una determinada zona. Posteriormente, el resto de jugadores lanzan una bola metálica (650-800 gr. de peso) e intentan dejarla lo más cerca posible del boliche. 
c) 1 Chavelho de 40 centímetros de altura.

d) 1 Chavelho de 50 centímetros de altura.

e) 1 Chavelho de 60 centímetros de altura.

- Distancia y disposición de cada elemento entre sí. En los cuatro Chavelhos en línea, la distancia entre cada uno será igual al de su tamaño en la horizontal, de forma que su simple caída no provoque un efecto dominó en los siguientes Chavelhos. En los otros diez Chavelhos, situados alrededor del Chavelho de 30 centímetros, el círculo será de 40 centímetros de diámetro (ver figura 3).

Figura 3 - Distancia y disposición de los 14 Chavelhos en el Parque das Portas do Corno do Bico.

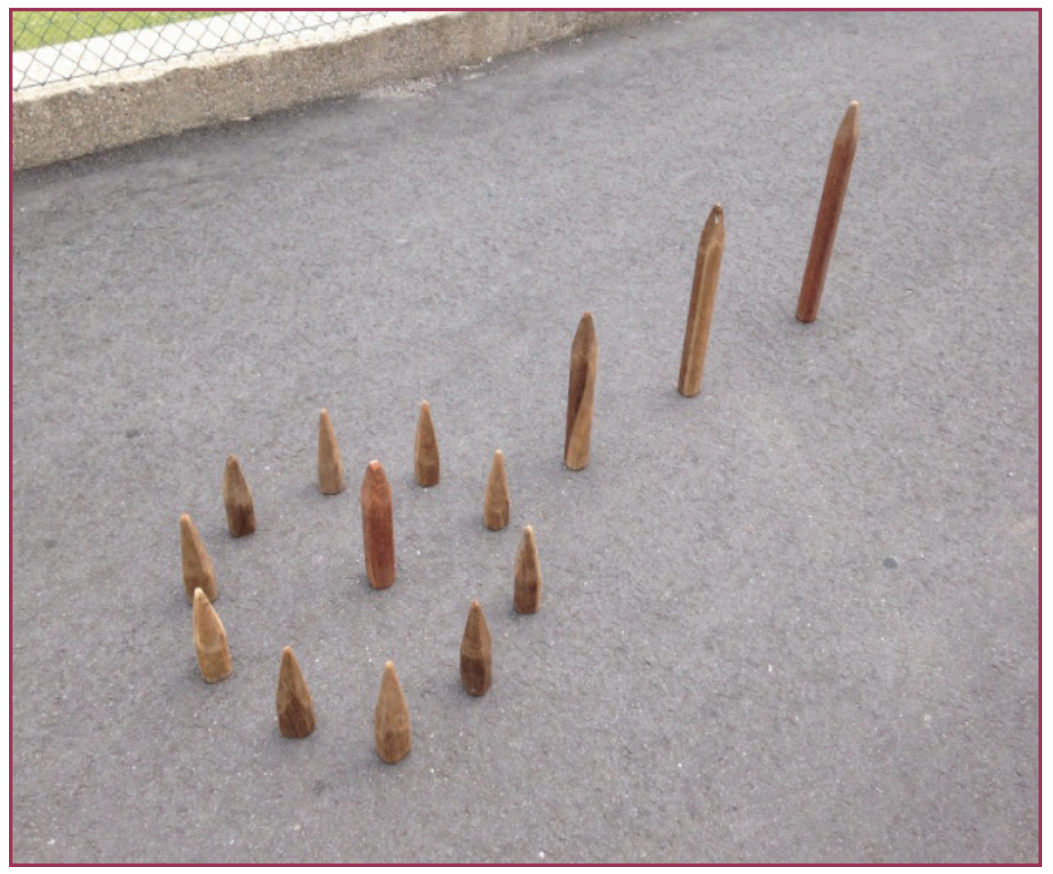

Fuente: Elaboración propia.

- Distancia de lanzamiento. El jugador efectuará el lanzamiento de la bola a 20 pasos en línea recta contados desde el Chavelho de 30 centímetros (central).

- Desarrollo del juego. Se puede jugar de forma individual o en parejas. El juego termina cuando se alcanzan los 1.000 puntos. Cada jugador tiene derecho al lanzamiento de dos bolas consecutivas, restableciendo los Chavelhos a su posición original después de cada lanzamiento. La puntuación de cada jugada será la suma de los puntos obtenidos por el lanzamiento de las dos bolas. El lanzamiento solo será válido si la bola rebasa el último Chavelho (60 centímetros).

- Puntuación. Cada Chavelho derribado tendrá una puntuación determinada conforme a su tamaño (ver figura 4).

\section{DISCUSIÓN}

El objetivo de este trabajo fue conocer y registrar las modalidades de juego de bolos que se manifiestan en el Camino Central Portugués, en la zona norte, que discurre por 9 municipios desde Porto hasta Valença do Minho. Realizada la investigación, vemos como el 
juego de bolos no es un juego popular y tradicional con arraigo en la zona norte de Portugal, salvo el caso particular del Jogo do Chavelho de Paredes de Coura y otros dos casos aislados que veremos a continuación.

Figura 4 - Puntuación según el tipo de Chavelho derribado.

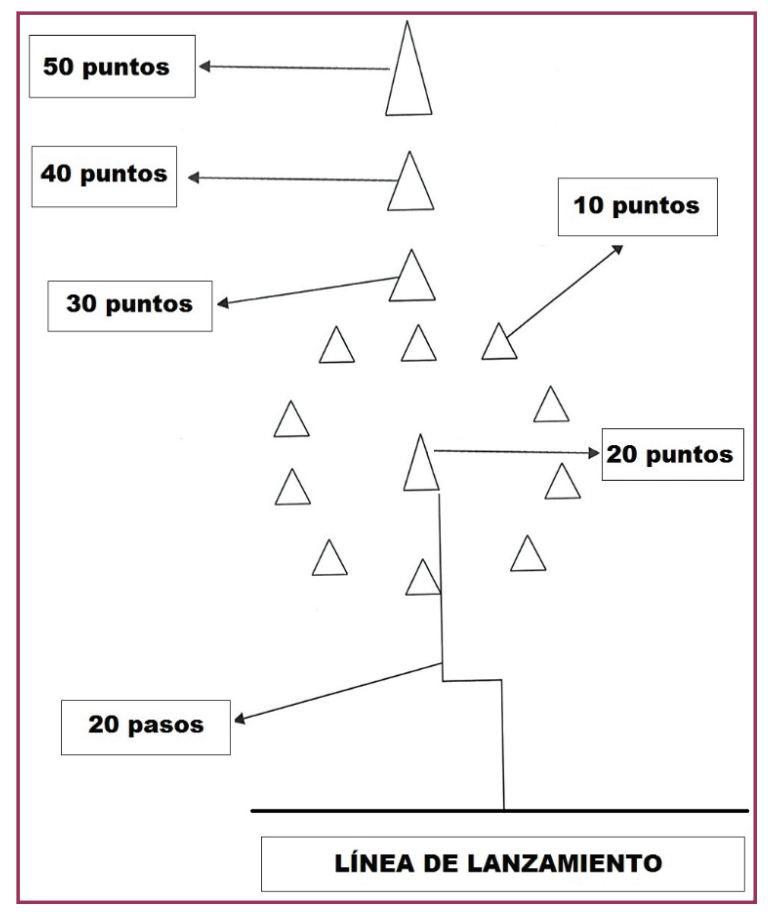

Fuente: elaboración propia.

Las tradiciones populares en esta zona portuguesa, al igual que ocurre en el resto del territorio gallego y español, han caído en desuso (SILVEIRA; CUNHA, 2014), dejando paso a la práctica de otras modalidades deportivas y recreativas más novedosas y modernas (MARIN et al., 2012; RODRIGUEZ; PEREIRA, 2016). Además, autores como Pérez y Tabernero (1997), Rodríguez, Pazos y Palacios (2015) o Romani (1979) añaden los cambios en la sociedad (fruto del progreso y de una modernización imparable) y la irrupción del gran deporte de masas como es el fútbol como causas también muy determinantes en el decaimiento de la práctica de juegos populares y tradicionales. Otros como Crespo (2012) relacionan este decaimiento de los juegos populares y tradicionales con el auge de las nuevas tecnologías 0, en palabras de Silva (2011, p. 144) con "[...] nuevas formas de socialización, de sociabilidad y nuevas formas culturales".

El juego de bolos es uno de los tipos de modalidades populares y tradicionales con más arraigo en España y Europa (CORRALES, 2010; GARCÍA, 1974; JIMÉNEZ, 2013; MORENO, 2008) pero esa popularidad y nivel de práctica no se corresponde con la zona estudiada en Portugal, en la que juegos como A Malha (CABRAL, 1998; NASCIMENTO et al., 2015; RIBAS, 2013) o la petanca (RODRÍGUEZ; PEREIRA, 2016) se muestran como actividades recreativas tradicionales con más arraigo en el país luso.

El juego de A Malha es posiblemente el juego popular y tradicional con más arraigo en Portugal. Pero, si observamos la tabla 1, vemos que el juego de petanca también tiene un alto nivel de práctica. Según las personas entrevistadas, esta circunstancia se debe principalmente a que es una actividad practicada por personas mayores (que no practican los llamados deportes modernos como fútbol, baloncesto... y no participan en actividades competitivas 
que requieren un considerable esfuerzo físico e intensidad en el juego) y que se trata de una actividad tradicional pero no típica de Portugal, sino de Francia; y esta actividad se practica en Portugal porque las personas retornadas de la inmigración en tierras francesas trajeron con ellos esa práctica ancestral del país.

En referencia al juego de bolos, se constata la práctica únicamente en Paredes de Coura, municipio por el que pasa el Camino del Norte Portugués del Camino de Santiago, tratándose de una modalidad de bolos de derribo que no se asemeja a ninguna de las practicadas en Galicia. Sin embargo, en otras zonas del norte de Portugal, se han localizado dos municipios que registran actividad bolística, como son los municipios de Bragança (en las parroquias o freguesías de Terroso, Alimonde, Conlelas, Carrazedo y Castrelos) y Vinhais (en freguesías como Tohiselo, Fresulfe, Vila Boa, Santa Cruz, Pazo de Vinhais y Vinhais).

Figura 5 - Disposición de los bolos de Vinhais y Bragança en la caja, con distintos tamaños de bolos.

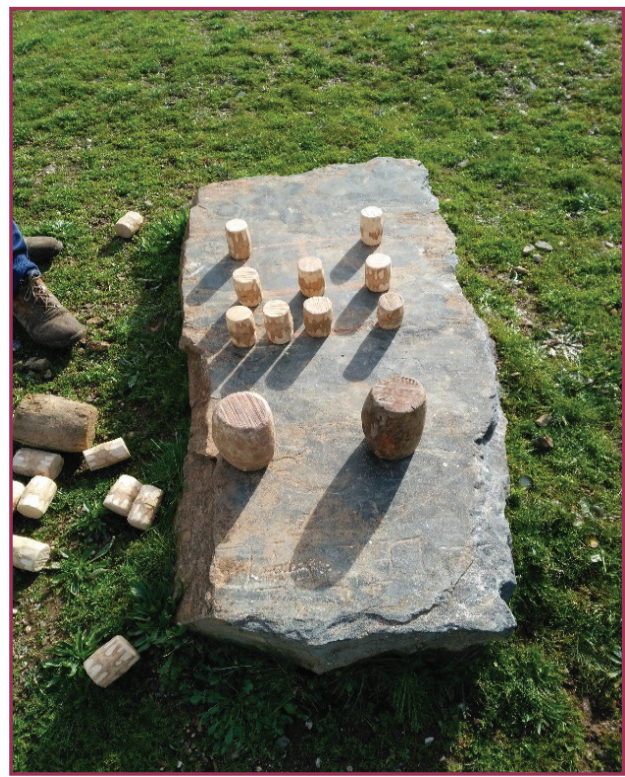

Fuente: Federación Gallega de Bolos

Figura 6 - Campo de juego de bolos en Bragança, donde se puede apreciar la caja de bolos en medio y, a ambos lados, las zonas de lanzamiento.

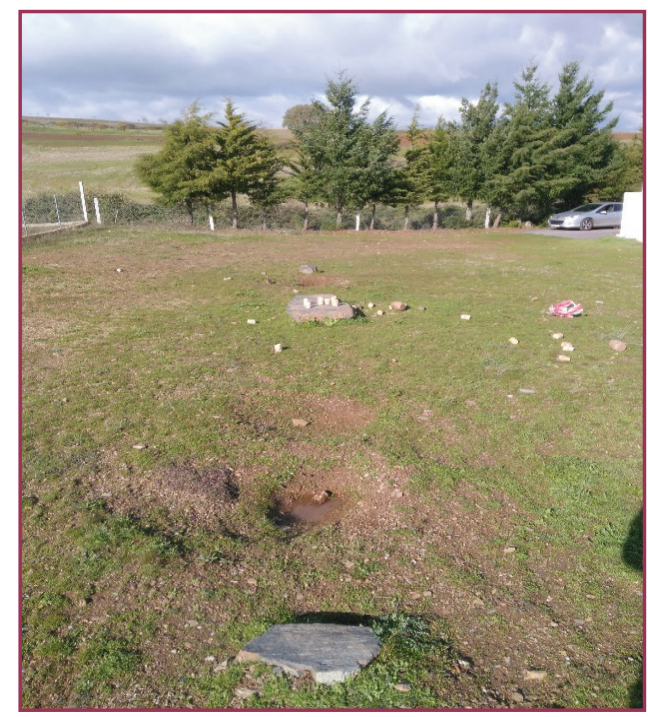

Fuente: Federación Gallega de Bolos. 
Figura 7 - Distinta disposición de los bolos en la caja, según la dirección de lanzamiento.

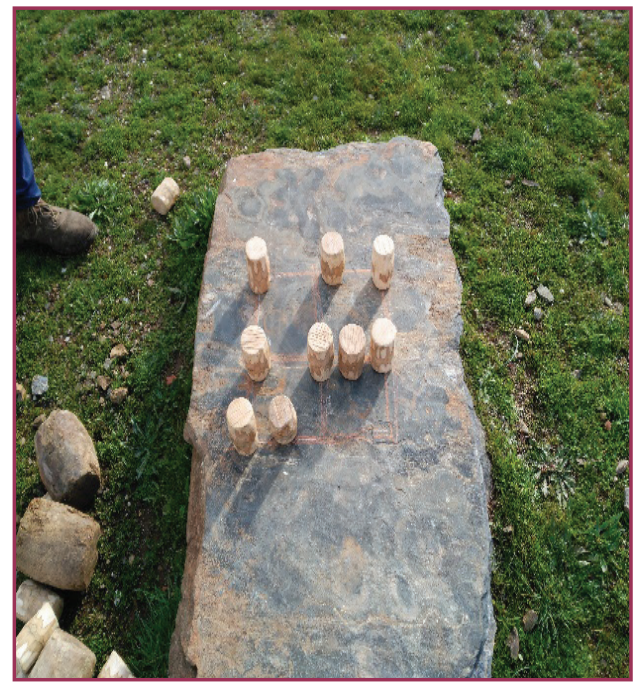

Fuente: Federación Gallega de Bolos.

Figura 8 - Campo de juego de bolos en el centro del pueblo (Carrazedo-Bragança), al lado de la iglesia.

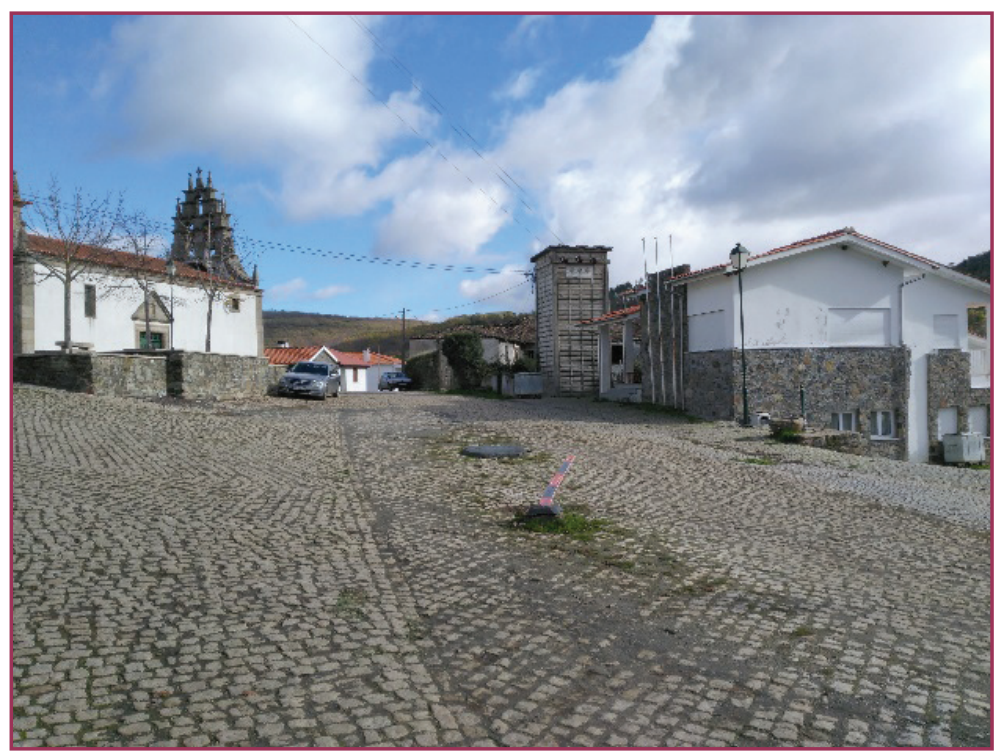

Fuente: Federación Gallega de Bolos.

En estas dos localidades portuguesas se practican modalidades semejantes a las practicadas en la otra parte de la frontera luso-gallega, en la provincia de Ourense (España), con bolos en forma de barril y descritos ya por autores como Rodríguez, Pazos y Palacios (2014b) pero que difieren en la modalidad (la portuguesa es de derribo, no de pasabolo) y variaciones en el número, tamaño, dirección de lanzamiento y distribución de los bolos en la caja (ver figuras 5, 6, 7 y 8).

El juego de bolos en la freguesía de Carrazedo (Bragança-Portugal) también se denomina, desde tiempos muy antiguos, Jogo dos Paus (DOS SANTOS-ROCA, 1985), como se puede comprobar en un mural situado en la casa del pueblo de la localidad (ver figura 9). Hoy en día, se mantiene el nombre del juego y el campo o largo para denominar el espacio de juego (ver figura 10). 
Figura 9 - Mural que hace referencia al juego de bolos (Jogo dos Paus) situado en una de las fachadas de la casa del pueblo de la freguesía de Carrazedo, en Bragança (Portugal).

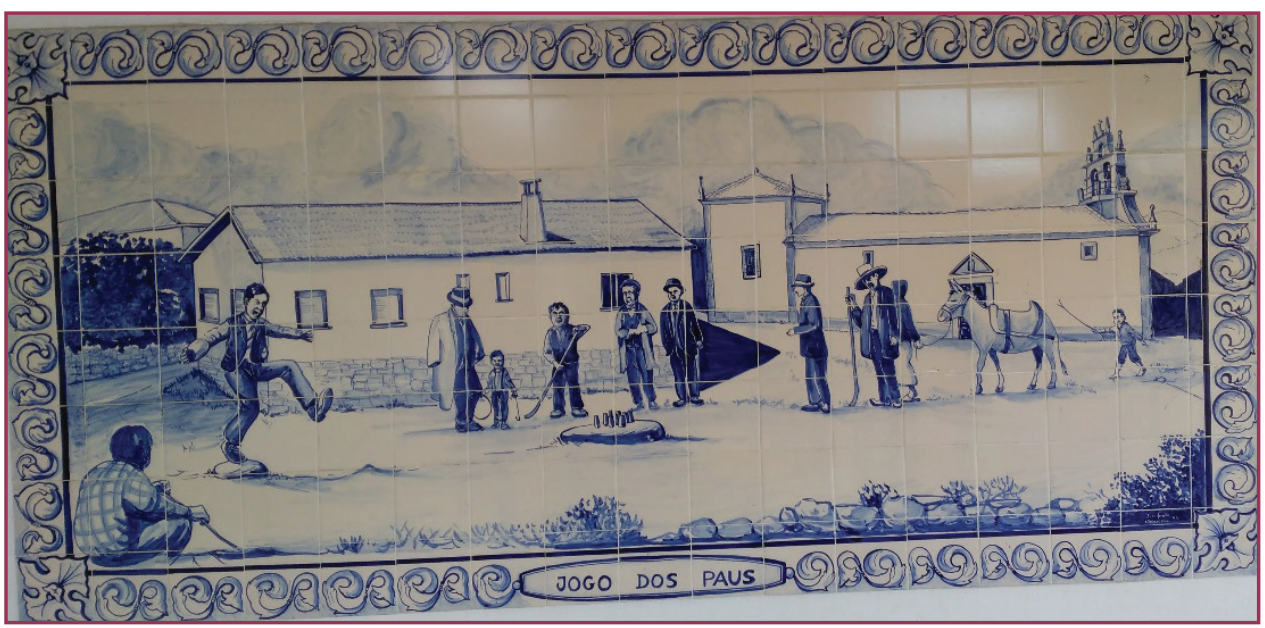

Fuente: Federación Gallega de Bolos.

Figura 10 - Referencia al campo de bolos o Jogo dos Paus situado en Carrazedo.

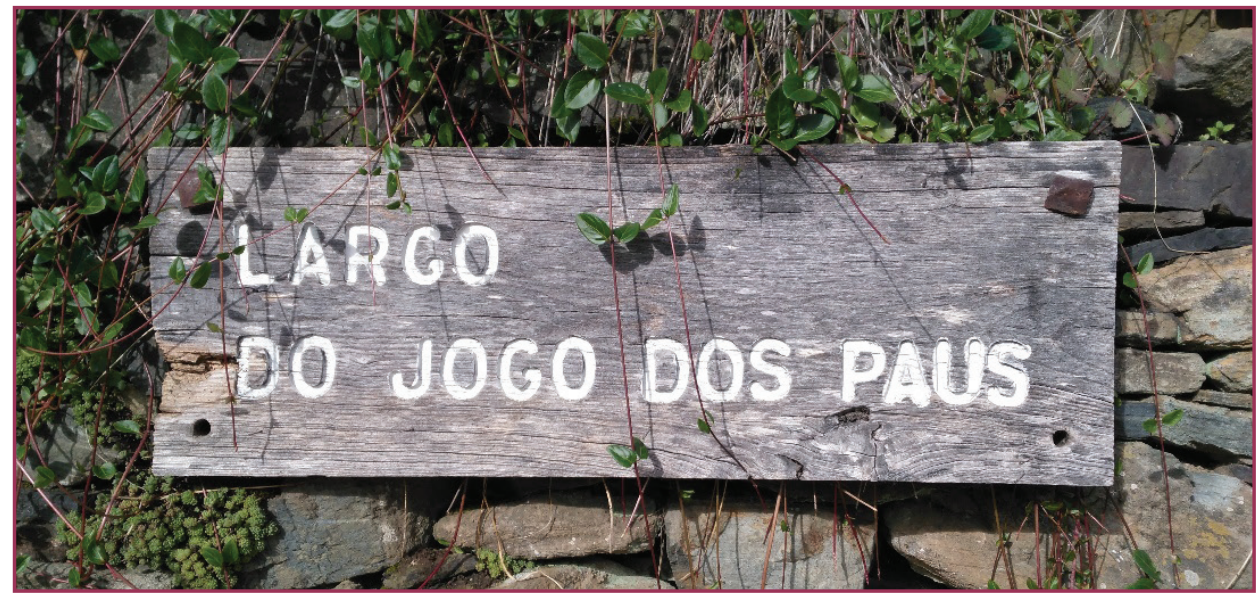

Fuente: Federación Gallega de Bolos.

El Camino de Santiago, auténtica vía de intercambio cultural, no se muestra en esta investigación como un determinante en la práctica del juego de bolos tradicional, como ocurre en las diferentes vías del Camino de Santiago a su entrada en Galicia, constatado en los estudios de Rodríguez, Pazos y Palacios (2014b).

Estos autores hacían referencia a las influencias germanas y francesas en el juego de bolos en la península española a través del Camino de Santiago; incluso Lavega (2013) hace referencia al intercambio de juegos y tradiciones a través de viajes culturales de portugueses, italianos o alemanes a otros países (como es el caso del patrimonio luso-brasileiro a través de la exportación del Jogo do Beto a la zona de Río Grande do Sul-Brasil). Pero, en este caso, el tramo estudiado del Camino de Santiago en Portugal no se ajusta a nuestra hipótesis de partida, donde se pensaba que la práctica bolística registrada en Galicia tendría su continuidad a la entrada del país luso.

Los participantes en el estudio destacan que los juegos populares y tradicionales no forman parte importante de los planes de estudio en Portugal, ni en la EB1 ni en la EB2. De hecho, los propios docentes indican que el uso que hacen de este tipo de juegos es como 
medio para conseguir otro tipo de objetivos motores o como introductorios de otro tipo de actividades principales en la sesión. Este hecho también es constatado por Bragada (2002) al referirse al juego popular y tradicional como medio y no como fin, alertando de que la escuela debería revisar sus programas educativos y buscar la forma de "[...] reavivar la mayoría de los juegos tradicionales" (p. 10).

Silveira y Cunha (2014) destacan la enorme potencialidad educativa de los juegos populares y tradicionales, que favorecen la aproximación entre diferentes generaciones e impulsan la imaginación. Sin embargo, también hacen referencia a las dificultades existenciales por las que pasan este tipo de prácticas en la actualidad, reflejando Silva (2011) que estas manifestaciones pasarán al olvido si no se realizan actuaciones concretas para evitarlo, añadiendo Crespo (2012) que sería necesario crear momentos y espacios específicos para la promoción y desarrollo de este tipo de juegos.

$Y$, cuando hablamos de espacios, hay que tener en cuenta que actualmente lo que se construye va en detrimento de las prácticas tradicionales, predominando modernos espacios deportivos con material específico de deportes de masas, pavimentos de hierba artificial, substitución de zonas verdes por novedosos parques infantiles... y, todo esto, condiciona en gran manera el tipo que práctica que se realiza en estos espacios (CALABUIG; QUINTANILLA; MUNDINA, 2008).

\section{CONSIDERACIONES FINALES}

La importante práctica (número de municipios donde se registra actividad, número de clubs de bolos, jugadores federados...) del juego de bolos tradicional registrado en el Camino de Santiago al sur de Galicia, cerca de la frontera con Portugal (RODRÍGUEZ; PAZOS; PALACIOS, 2016), no tiene su continuidad en el país luso, pues en el Camino Central Portugués, en la zona norte que comprende nueve municipios, de Porto a Valença do Minho, tan sólo se registra actividad en el municipio de Paredes de Coura, a través del Jogo do Chavelho.

A pesar de que en la zona norte portuguesa, en los municipios de Vinhais y Bragança (fuera estos del Camino de Santiago) también se registra actividad bolística, este juego popular y tradicional, para muchos el más practicado en el mundo en sus diferentes modalidades y variantes, y en base a los datos obtenidos en ese estudio, no tiene arraigo en la zona norte de Portugal. El juego de A Malha su principal actividad tradicional, practicada hoy en día en todos los municipios analizados por hombres mayores, aspecto típico también del juego de bolos y, en general, de los juegos populares y tradicionales que implican un cierto componente físico (RODRÍGUEZ, 2016) y en el que la mujer siempre ha tenido un papel secundario (RODRíGUEZ; PAZOS; PALACIOS, 2016).

En los centros educativos portugueses el juego popular y tradicional no constituye un bloque de contenidos importante en el currículum. Los docentes emplean este tipo de juegos como medio y no como fin, restándole mucha de la importancia que tienen estas prácticas, válidas más allá que como mero recurso para realizar un calentamiento en una clase de Educación Física.

El conocimiento de la cultura propia del país, el acercamiento entre generaciones, el culto a las propias tradiciones, el respecto por el pasado, son aspectos que se pueden trabajar 
a través del juego popular y tradicional y que hoy en día está quedando en el olvido, bien por la dejadez de las autoridades políticas y educativas (que deberían preservar la cultura tradicional a través de programas formativos y educativos) y, también, por la amplia oferta lúdica, deportiva y tecnológica que hoy en día impera en nuestra sociedad; una sociedad consumista que reniega del pasado como forma de construir un futuro diferente.

\section{LIMITACIONES Y PERSPECTIVA DE FUTURO}

El presente estudio se limita al análisis de la última parte del Camino Central Portugués (Porto-Valença do Minho). Estudios similares a este, que propongan el análisis de las prácticas tradicionales en el resto del Camino Central Portugués (de Lisboa a Porto) nos dará una visión general del tratamiento de los juegos populares y tradicionales en Portugal, aspecto que nos debe servir para valorar en su justa medida la más que interesante cultura lúdica portuguesa.

\section{REFERENCIAS}

ALONSO, Jesús Alberto et al. Propuesta didáctica bilingüe para Educación Física en Educación Primaria. Juegos populares y tradicionales ingleses. Revista Retos, Nuevas Tendencias en Educación Física, Deportes y Recreación, n.28, p.116-121, jul. 2015.

AMADO, João. Manual de investigação qualitativa em educação. Coimbra: Coimbra University, 2014.

ANDREU, Eliseo. Traditional children's games in the mediterranean: anologies. Journal of Human Sport \& Exercise, v.4, n.3, p.201-210, oct. 2009.

ARAÚJO, Paulo Coelho de et al. O jogo tradicional como patrimonio cultural Luso-Brasileiro. O caso do jogo Beto/Betes. In: MARÍN, Elizara Carolina; RIBAS, João Francisco Magno. Jogo tradicional e cultura. Santa María: Editora UFSM, 2013. p.235-273.

BAPTISTA, Iraê. Metodologia de pesquisa em Ciências Sociais. Maputo-Moçambique: Escolar Editora, 2016.

BOGDAN, Robert; BIKLEN, Sari. Investigação qualitativa em educação: uma introdução à teoria e aos métodos. Porto: Porto Editora, 2013.

NASCIMENTO, Thaiane Bonaldo do et al. Lógica interna e contexto cultural: relações a partir de jogos tradicionais. Revista Cinergis, v.16, n.4, p.312-318, oct./dez. 2015.

BRAGADA, José. Jogos tradicionais e o desenvolvimento das capacidades motoras na escola. Lisboa: Centro de Estudos e Formação Desportiva, 2002.

CABRAL, António. Jogos populares portugueses. Porto: Domingos Barreira, 1998.

CALABUIG, Ferrán; QUINTANILLA, Ismael; MUNDINA, Javier. La calidad percibida de los servicios deportivos: diferencias según instalación, género, edad y tipo de usuario en servicios náuticos. Revista Internacional de Ciencias del Deporte, v.4, n.10, p.25-43, ene. 2008. 
CORRALES, Antonio Rafael. El juego de bolos en la filatelia. Revista digital Educación Física y Deportes, n.141, feb., 2010. Disponible en: <http://www.efdeportes.com/efd141/el-juego-de-losbolos-en-la-filatelia.htm>. Acceso en: 1․ mayo 2014.

CRESPO, Jorge. 0 espírito do jogo: estudos e ensaios. Lisboa: Colibri, 2012.

DENZIN, Norman; LINCOLN, Yvonna. Manual de investigación cualitativa (V.IV). Métodos de recolección y análisis de datos. Barcelona: Gedisa, 2015.

DIAS, Gonçalo; MENDES, Rui. Jogos tradicionais portugueses. Retrospectiva e tendencias futuras. Revista Exedra, n.3, p.51-58, 2010.

DOS SANTOS-ROCA, Valdemar. O jogo dos paus em Carrazedo. Trabalhos de Antropologia e Etnología, v.25, n.1, p.121-134, 1985.

ESCOBAR-PÉREZ, Jazmine; CUERVO-MARTÍNEZ, Ángela. Validez de contenido y juicio de expertos: una aproximación a su utilización. Avances en Medición, n.6, p.27-36, 2008. Disponible en: <http://www.humanas.unal.edu.co/psicometria/files/7113/8574/5708/Articulo3 Juicio de expertos 27-36.pdf>. Acceso en: 19 mayo 2018.

FLICK, Uwe. El diseño de investigación cualitativa. Madrid: Morata, 2015.

GARCÍA, Rafael. Juegos y deportes tradicionales de España. Textos de las Cátedras Universitarias de Tema Deportivo Cultural de la Universidad de Navarra, n.14, p.54-111, 1974.

HERNÁNDEZ, José et al. Análisis diacrónico de la comunicación motriz de los juegos y deportes tradicionales canarios. Revista Internacional de Medicina y Ciencias de la Actividad Física y el Deporte, v.15, n.57, p.123-137, 2015.

JIMÉNEZ, Enrique. El juego de bolos de Abades, patrimonio cultural y deportivo de Segovia (Deportes Autóctonos de Castilla y León). Revista digital Educación Física y Deportes, n.186, nov., 2013. Disponible en: <http://www. efdeportes.com/efd186/el-juego-de-bolos-de-abades-desegovia.htm>. Acceso en: 15 ab. 2014.

LARA, Amador José. Transmission of social and educational values through sport. Journal of Sport and Health Research, v.3, n.1, p.5-6, jan./apr. 2011.

LAVEGA, Pere. Posfácio. In: MARÍN, Elizara Carolina; RIBAS, João Francisco Magno. Jogo tradicional e cultura. Santa María: Editora UFSM, 2013. p.297-301.

LAVEGA, Pere; NAVARRO, Vicente. La motricidad en los juegos de Rodrigo Caro: días geniales o lúdicos (1626). Revista Internacional de Medicina y Ciencias de la Actividad Física y el Deporte, v.15, n.59, p.489-505, sep. 2015.

LIMA, Leonor; PALHARES, José Augusto. Metodologia de investigação em Ciências Sociais da Educação. Braga: Humus, 2014.

MARIN, Elizara Carolina et al. Jogos tradicionais no Estado do Rio Grande do Sul: manifestação pulsante e silenciada. Movimento, v.18, n.3, p.73-94, jul./set. 2012.

MÉNDEZ-GIMÉNEZ, Antonio; FERNÁNDEZ-RÍO, Javier. Análisis y modificación de los juegos y deportes tradicionales para su adecuada aplicación en el ámbito educativo. Revista Retos, Nuevas Tendencias en Educación Física, Deportes y Recreación, n.19, p.54-58, ene. 2011. 
MORENO, Cristóbal. Juegos y Deportes Tradicionales de España. Madrid: Sociedad Estatal de Correos y Telégrafos, 2008.

PARLEBAS, Pierre. Juegos, deporte y sociedad. Léxico de praxiología motriz. Barcelona: Paidotribo, 2001.

PARLEBAS, Pierre. Prefácio. In: MARÍN, Elizara Carolina; RIBAS, João Francisco Magno. Jogo tradicional e cultura. Santa María: Editora UFSM, 2013. p.11-18.

PÉREZ, Ricardo; TABERNERO, Xaquín Alberte. Juegos populares en Galicia. Santiago: Lea, 1997.

POMBO, Antón. Cuaderno de peregrino-Camino Portugués. Madrid: Anaya, 2012.

PONTES, Victoria. El patrimonio vivo en el museo. In: BELLIDO, María Luisa. Arte y museos del siglo XXI: entre los nuevos ámbitos y las inserciones tecnológicas. Barcelona: UOC, 2014. p.73-94.

QUIVY, Raymond; CAMPENHOUDT, Luc Van. Manual de investigação em Ciências Sociais. Trajectos. Lisboa: Gradiva, 2017.

RAMOS, César. Visiones del Camino de Santiago desde el medievalismo español. Revista AGALI, v.1, n. 1, p.1-24, ene. 2011.

REBOLLO, José Antonio. Juegos populares: una propuesta para la escuela. Revista Retos, Nuevas Tendencias en Educación Física, Deportes y Recreación, n.3, p.31-36, sep. 2002.

RIBAS, João Francisco Magno. Grupo Social Português. In: MARÍN, Elizara Carolina; RIBAS, João Francisco Magno. Jogo tradicional e cultura. Santa María: Editora UFSM, 2013. p.205-231.

RODRÍGUEZ DE LA CRUZ, Julio César. Juegos del S. XVI en las clases de Educación Física de Secundaria. Revista Retos, Nuevas Tendencias en Educación Física, Deportes y Recreación, n.13, p.50-55, ene. 2008.

RODRÍGUEZ, José Eugenio. 0 estudo do xogo dos bolos en Boiro: aspectos históricos, estado actual de práctica e perspectivas de futuro. 2013. $494 \mathrm{f}$. Tesis (doctorado) - A Coruña: Universidade da Coruña, 2013.

RODRÍGUEZ, José Eugenio. 0 estudo do xogo dos bolos en Boiro: cultura, tradición e educación. A Coruña: Toxosoutos, 2016.

RODRÍGUEZ, José Eugenio; PAZOS, José María; PALACIOS, José. El juego de bolos de Boiro: la necesidad de reglamentación de una modalidad de pasabolo atípica en España. Revista Digital EmásF, n.28, p.17-43, mayo/jun. 2014a.

RODRÍGUEZ, José Eugenio; PAZOS, José María; PALACIOS, José. Costumbres lúdicas en España: el juego de bolos en la etapa final del Camino de Santiago. Movimento, v.20, n.4, p.1397-1421, out./dez. 2014b.

RODRÍGUEZ, José Eugenio; PAZOS, José María; PALACIOS, José. Causas del decaimiento de la práctica del juego de bolos en Boiro. Revista Internacional de Deportes Colectivos, n.23, p.80-83, 2015.

RODRÍGUEZ, José Eugenio; PAZOS, José María; PALACIOS, José. El rol desempeñado por la mujer en el juego de bolos de Boiro. Movimento, v.22, n.3, p.971-984, jul./set. 2016. 
RODRÍGUEZ, José Eugenio; PEREIRA, Beatriz. Práctica de juegos tradicionales en el Camino Central Portugués. Transferencia al ámbito escolar. In: CONGRESO MUNDIAL DEL DEPORTE ESCOLAR. EDUCACIÓN FÍSICA Y PSICOMOTRICIDAD. 6., 2016. [Anales]...A Coruña:

Universidade da Coruña, 2016. E12, p.1-13.

ROMANI, Arturo. Xogos infantiles de Galicia. Santiago: Follas Novas, 1979.

RUIZ, José Gerardo. Estudios de los bolos en Asturias: aspectos histórico-culturales, modalidades, elementos y materiales de juego. Estado actual de su práctica. 2000. $440 \mathrm{f}$. Tesis (doctorado) - Granada: Universidad de Granada, 2000.

SÁNCHEZ, Jorge. El camino portugués a Santiago. Madrid: Dilema, 2010.

SILVA, Alberto Nídio. Jogos, brinquedos e brincadeiras: trajectos intergeracionais. Vila Verde: ATAHCA, 2011.

SILVEIRA, Luciene; CUNHA, António Camilo. 0 jogo e a infância: entre o mundo pensado e 0 mundo vivido. Santo Tirso: Whitebooks, 2014.

TORRE, Carlos. El Bolo Palma: de Trasmiera a Vizcaya. Beranga-Cantabria: Quinzaños, 2013.

VEIGA, Francisco. Xogo popular galego e educación: Vixencia educativa e función de identificación cultural dos xogos e enredos tradicionais. 1998. 624 f. Tesis (doctorado) - Santiago de Compostela: Universidad de Santiago de Compostela, 1998.

VIGNE, Mickaël. Las actividades tradicionales de ocio como reflejo de una sociedad. Revista Científica Digital Acción Motriz, n.7, p.62-76, jul./dic. 2011. 
